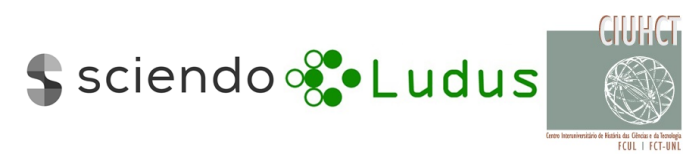
Articles

\title{
New Year Mathematical Card or V Points Mathematical CONSTANT
}

\author{
Valery Ochkov \\ National Research University Moscow Power Engineering Institute \\ ochkov@twt.mpei.ac.ru
}

\begin{abstract}
The article describes an attempt to define a new mathematical constant - the probability of obtaining a hyperbola or an ellipse when throwing five random points on a plane.
\end{abstract}

Keywords: second-order plane curve, hyperbola, ellipse, Mathcad, pseudo-random number.

The author has such a long pre-New Year's habit. He and his students develop and post on the site of the mathematical package [1] greeting animated cards with some entertaining mathematical meaning.

At the end of 2017, such New Year's postcard was published: in a square on a plane, five points are randomly scattered 1000 times, through which a second-order plane curve was drawn. All who recognize all the seven possible curves on this postcard (seven is a beautiful number!), could expect happiness and luck in the New Year [2, 3] $]^{1}$.

The prehistory of this New Year's postcard is as follows. In one lesson with his students, the author showed a sagging chain (see Figure 1) and asked a question, the graph of what function this all resembles. The students answered in chorus that it was a parabola 4 . Then the coordinates of five points (left, right, bottom and two points in the middle of the "branches" of the chain) were taken from the photograph of the chain in the environment of the graphic editor Paint, through which a plane curve of the second order was drawn. These five points were taken from the photographs of the sagging chain in different positions and with varying degrees of tension. After processing the data, it turned out that the curve of the second order passing through the five "chain" points was, of course, not a parabola and not even one of the branches of the

\footnotetext{
${ }^{1}$ On the site ww. demonstrations.wolfram.com/FivePointsDetermineAConicSection we can see only two curves.
} 
hyperbola, but... an arc of an ellipse. The lesson ended with the description of the function of the chain line (a catenary), which more or less accurately (taking into account measurement errors) passes through three, four, five and more points "taken" from the photograph of the sagging chain.

A question arose: which second-order curve passes through five randomly chosen points on a plane? Having at hand a computer with the program Mathcad [5], it can be tried to determine that by statistical tests (Monte Carlo) 6] .
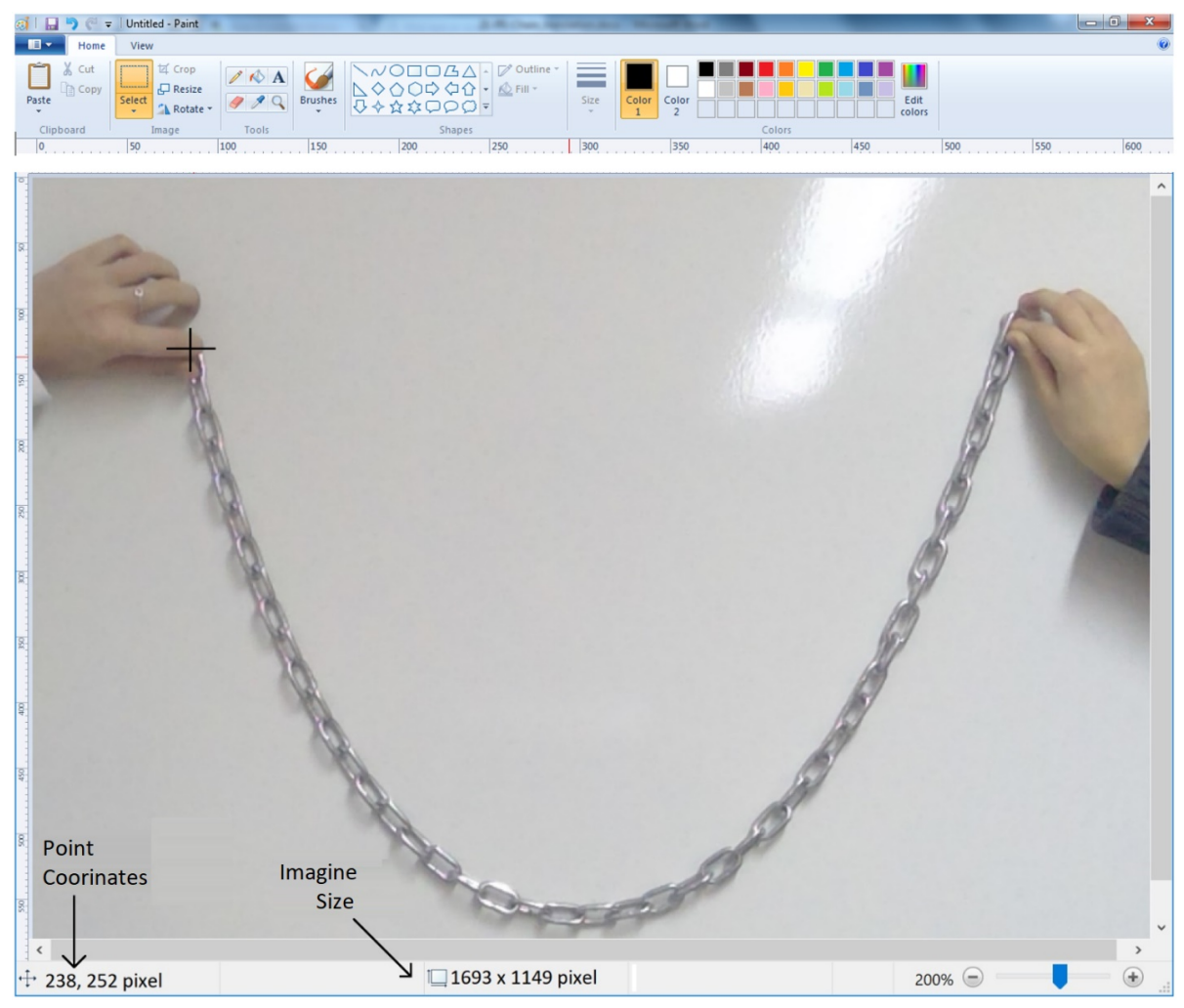

Figure 1: Experiment with a sagging chain.

It is known that through five points on the plane one can draw the following curves [2]:

1. Two branches of a hyperbola;

2. An ellipse;

3. A parabola (transitional case from hyperbola to ellipse);

4. A circle (special case of an ellipse);

5. Two intersecting lines (degenerate case of two branches of a hyperbola);

6. Two parallel straight lines;

7. One straight line (a special case of cases 5 and 6). 
In practice (not in theory), five randomly points can hold only two curves: a hyperbola with two branches and an ellipse (Figure 22. The author and his students "drew" manually the other five curves on the New Year's card, asking the "exact" coordinates of five points.

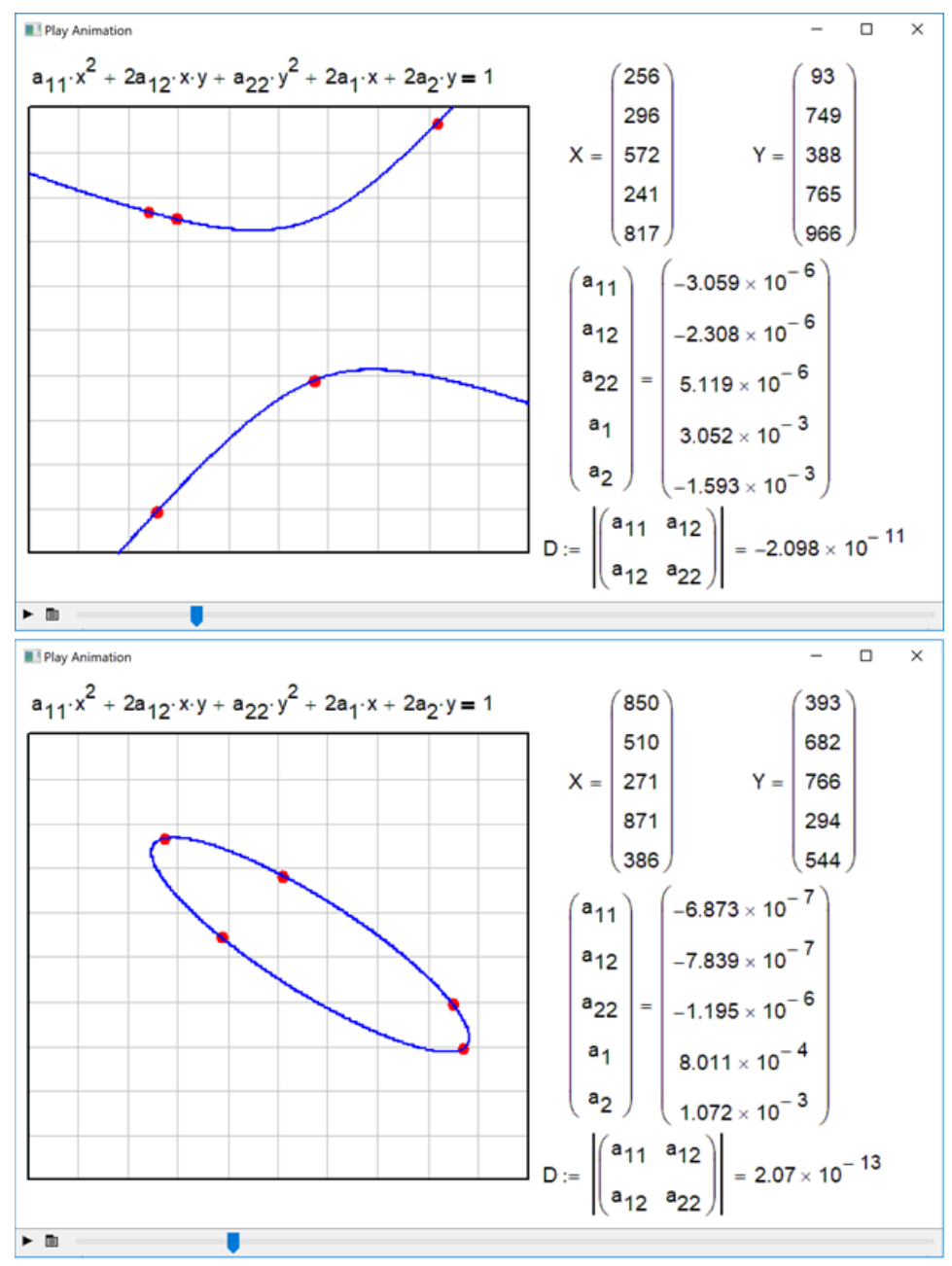

Figure 2: A hyperbola (above) and an ellipse drawn through five random points.

Figure 2 shows two frames of the New Year's animation card with a hyperbola and an ellipse. The coordinates of the five random points (the values of the vectors $X$ and $Y$ ) were also shown on the card, the values of the five coefficients of the second-order curve equation $\left(a_{11}, a_{12}, a_{22}, a_{1}\right.$, and $\left.a_{2}\right)$ : see the equation on the top of Figure 2), as well as the values of the invariant $D$ over which the curve was determined: $D<0$ is a hyperbola and $D>0$ is an ellipse [2].

For $D=0$ (and under other additional conditions), a parabola (the transient case from the ellipse to the hyperbola) must be obtained. But that case, we emphasize, took place only with an artificial, and not random assignment of the values of the vectors $X$ and $Y$. 
Those who received the New Year card were asked to calculate how many times they saw a hyperbola, and how many times they saw an ellipse (for "full happiness" in the coming 2018).

It turned out that the hyperbola appeared in approximately $71.84 \%$ of the cases, and the ellipse in the remaining $28.16 \%$. This was calculated, of course, not through viewing animation frames, but through a statistical computer experiment (Figure 3].

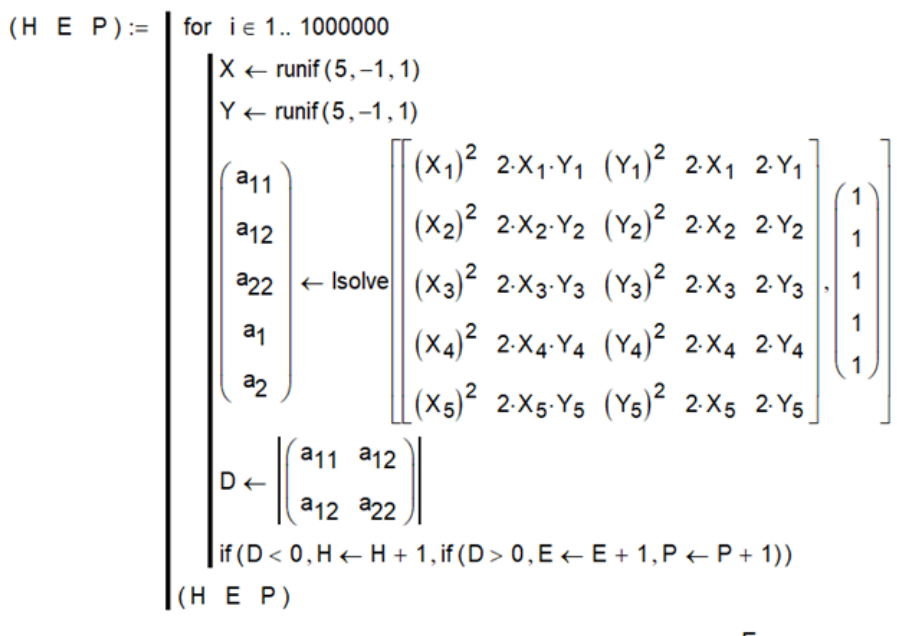

$$
\begin{aligned}
& H=719484 \quad E=280516 \quad P=0 \quad H+E+P=1000000 \quad \frac{E}{H}=0.389885
\end{aligned}
$$

Figure 3: Ocurrences of hyperbolas and ellipses - Mathcad layout.

Figure 3 shows the number of dropped hyperbolas $(\mathrm{H})$ and ellipses $(\mathrm{E})$ when throwing five random points ten million times in a square of $2 \times 2$. At the same time (just in case!), the number of dropped parabolas $(\mathrm{P})$ was counted.

Regarding Mathcad layout (Figure 3), it suffices to explain the essence of the following operators and functions:

1. The FOR command makes a loop parameter i (throwing points into a square);

2. The function RUNIF generates 5 numbers (the first argument of the function) with a random (pseudo-random) distribution in the interval from -1 to 1 (the second and third arguments of the RUNIF function);

3. The LSOLVE function returns the solution of the system of linear algebraic equations (SLAE), the coefficient matrix for the unknowns is the first argument of the LSOLVE function, and the vector of the free terms is the second argument. The SLAE solutions is the vector of the coefficients of the required second-order equation $\left(a_{11}, a_{12}, a_{22}, a_{1}\right.$ and $\left.a_{2}\right)$;

4. The IF function counts the "dropped" hyperbolas, ellipses and parabolas. 
At the bottom of Figure 3 it is shown that, with ten million tossing, the five points of the hyperbola (H) fell 719484 times, the ellipse (E) 280516 times, and parabola $(\mathrm{P})$, as expected, never. The same approximate figures are obtained with other amounts of casts, and with squares of different sizes, where five points were thrown. We'll discuss the problematic of the shape of the region later.

Many people, after receiving a greeting card, read the message, admire the picture and...throw a postcard into a desk drawer or even throw it away. The publication on the Mathcad user forum of the "New Year" postcard with hyperbolas and ellipses had other consequences:

1. It was suggested that the new mathematical constant of 0.2806 should have a name: V Points 2 . One colleague of the author from the department of higher mathematics asserts that he has found an analytical way to calculate this constant, but he did not have time to write that down ${ }^{3}$

2. One visitor of the forum, Frank Purcell from Chicago, suggested that this constant can be determined (estimated) in another way. When solving the problem of four points (Sylvester's Four-Point Problem ${ }^{4}$ ), through which two intersecting parabolas are held, breaking a square area into certain zones [7, the fifth random point can fall into one of these zones, determining if the curve through the five points is a hyperbola or a parabola. The sum of the areas of these zones gives our constant. Sylvester's Four-Point Problem is described on [8].

3. At the forum of Mathcad, a race started - after throwing some points into the plane the idea was to draw through them a curve of higher order (Figure 4).

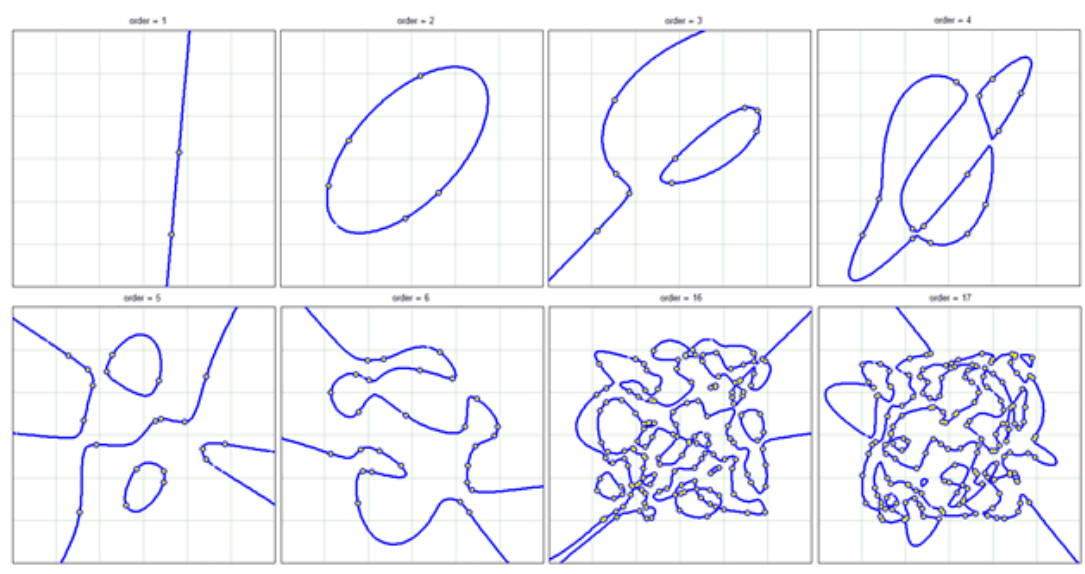

Figure 4: Curves of different orders.

\footnotetext{
${ }^{2} \mathrm{~V}$ Points - Google translator gives the Russian author's name В Очков.

${ }^{3}$ This man wants to be like Pierre Fermat, who wrote down his great theorem, but did not give a proof. Only a few centuries later a proof was found.

${ }^{4}$ Four points are randomly dashed to the plane and it is determined whether they form a convex quadrangle or one of the points is inside the triangle formed by the other three points.
} 
4. If the shape of the region is not a square, but a circle, then a slightly different value for the $\mathrm{V}$ Points constant is obtained (Figure 5 ). What is its value?

$$
\begin{aligned}
& N:=10^{6} \quad r:=3 \\
& \left(\begin{array}{l}
H \\
E
\end{array}\right):=\text { for } i \in 1 . . \mathrm{N} \\
& \text { E }:=\quad \begin{array}{r}
\text { for } i \in 1 \ldots N \\
\mid \text { while } 1
\end{array} \\
& \mid \begin{array}{l}
X \leftarrow \operatorname{runif}(5,-r, r) \\
Y \leftarrow \operatorname{runif}(5,-r, r)
\end{array} \\
& \mathrm{F} \leftarrow 0 \\
& \text { for } i \in 1 . .5 \\
& F \leftarrow F+1 \text { if }\left(X_{i}\right)^{2}+\left(Y_{i}\right)^{2}>r^{2} \\
& \text { break if } \mathrm{F}=0 \\
& \left.\left(\begin{array}{l}
a_{11} \\
a_{12} \\
a_{22} \\
a_{1} \\
a_{2}
\end{array}\right) \leftarrow \text { Isolve }\left[\begin{array}{lllll}
\left(X_{1}\right)^{2} & 2 \cdot X_{1} \cdot Y_{1} & \left(Y_{1}\right)^{2} & 2 \cdot X_{1} & 2 \cdot Y_{1} \\
\left(X_{2}\right)^{2} & 2 \cdot X_{2} \cdot Y_{2} & \left(Y_{2}\right)^{2} & 2 \cdot X_{2} & 2 \cdot Y_{2} \\
\left(X_{3}\right)^{2} & 2 \cdot X_{3} \cdot Y_{3} & \left(Y_{3}\right)^{2} & 2 \cdot X_{3} & 2 \cdot Y_{3} \\
\left(X_{4}\right)^{2} & 2 \cdot X_{4} \cdot Y_{4} & \left(Y_{4}\right)^{2} & 2 \cdot X_{4} & 2 \cdot Y_{4} \\
\left(X_{5}\right)^{2} & 2 \cdot X_{5} \cdot Y_{5} & \left(Y_{5}\right)^{2} & 2 \cdot X_{5} & 2 \cdot Y_{5}
\end{array}\right],\left(\begin{array}{l}
1 \\
1 \\
1 \\
1 \\
1
\end{array}\right]\right) \\
& D \leftarrow\left|\left(\begin{array}{ll}
a_{11} & a_{12} \\
a_{12} & a_{22}
\end{array}\right)\right| \\
& \text { if }(D<0, H \leftarrow H+1, E \leftarrow E+1) \\
& \left(\begin{array}{l}
\mathrm{H} \\
\mathrm{E}
\end{array}\right) \\
& H=701742 \\
& E=298258 \\
& \frac{\mathrm{H}}{\mathrm{N}}=0.70174 \quad \frac{\mathrm{E}}{\mathrm{N}}=0.29826
\end{aligned}
$$

Figure 5: Hyperbolas and ellipses in a circular region.

By the way, the students were also asked about an impression: is a plane an infinite extension of a square or a circle? Again, without thinking aabout the contours of such a question, they answered in chorus that it was an infinite extension of a square. That is understandable - in school, they are used to the Cartesian coordinate system, but have little contact with polar, cylindrical or spherical coordinates. The lesson mentioned at the beginning of this article ended with a story about how the function describing the chain line (a catenary) was independently and almost simultaneously discovered by Bernoulli, Huygens and Leibniz [9]. 


\section{Final Remarks}

This mathematical study is included in a book that is being prepared for publication by CRC/Tailor \& Francis with the title " $2^{5}$ Problems for STEM Education" [5]. The book introduces a new and emerging course for undergraduate STEM programs called "Physical-Mathematical Informatics", following a new direction in education called STE(A)M (Science, Technology, Engineering, [Art] and Mathematics). The book is for undergraduate students (and high school students), teachers of mathematics, physics, chemistry and other disciplines (humanities), and readers who have a basic understanding of mathematics and math software 5 .

\section{References}

[1] ] Ochkov V. F., Chudova J. V., Sokolov, A. V. "Using the PTC Community/Mathcad Forum in Computer Science Classes", IT in Schools, 10, 46-51, 2015.

http://twt.mpei.ac.ru/ochkov/PTC-Community-Lyc.pdf https://community.ptc.com/t5/PTC-Mathcad/ct-p/PTCMathcad

[2] Weisstein E. W. "Plane Curve", from MathWorld-A Wolfram Web Resource. http://mathworld.wolfram.com/PlaneCurve.html

[3] https://community.ptc.com/t5/PTC-Mathcad-Questions/ New-2018-Year/m-p/495771

[4] Ochkov V. F., Tsurikov G. N., Chudova J. V. "Caution: A Catanary", IT in Schools, 4, 58-62, 2017. http://twt.mpei.ac.ru/ochkov/Chain.pdf

[5] Ochkov V. F, Bogomolova E. P., Ivanov D. A. Physical and Mathematical Informatic with Mathcad and Internet, Moscow, Lan Publishing House, 556 pp, 2018. http://twt.mpei.ac.ru/ochkov/T-2018/PhysMathStudies.pdf English version ( $2^{5}$ Problems for STEM Education): https://www . crcpress.com/25-Problems-for-STEM-Education/ Ochkov/p/book/9780367345259

[6] Monte Carlo Method: https://en.wikipedia.org/wiki/Monte_Carlo_ method

[7] MATHCAD forums. https://community.ptc.com/t5/PTC-Mathcad- Questions/Firecrackers- 2018- or- 5 - th- order-curve- and- 20 - points/m-p/496511 ht tps ://community.ptc.com/t5/PTC-Mathcad-Questions/Hyperbola- and-E1lipse- new-math- constant/td-p/495992

[8] Sylvester's Four-Point Problem: http://mathworld.wolfram.com/SylvestersFour-PointProblem.html

[9] Ochkov V. F., Ochkova N. A. "The project of the monument to three mathematicians or MathMetria", Cloud of Science, 4, 548-571, 2017. http://twt.mpei.ac.ru/ochkov/MathMetria.pdf

\footnotetext{
${ }^{5}$ https : //www . crcpress . com/25-Problems-for-STEM-Education/0chkov/p/book/ 9780367345259 